\title{
Comprehensive chromosomal aberrations in a case of a patient with TCF3-HLF- positive BCP-ALL
}

\author{
Monika Lejman ${ }^{1 *}$ (D), Monika Włodarczyk² ${ }^{2}$, Joanna Zawitkowska ${ }^{3}$ a and Jerzy R. Kowalczyk ${ }^{3}$ (D)
}

\begin{abstract}
Background: The use of high-throughput analytical techniques has enabled the description of acute lymphoblastic leukaemia (ALL) subtypes. The TCF3-HLF translocation is a very rare rearrangement in ALL that is associated with an extremely poor prognosis. The TCF3-HLF fusion gene in the described case resulted in the fusion of the homeoboxrelated gene of TCF3 to the leucine zipper domain of HLF. The TCF3-HLF fusion gene product acts as a transcriptional factor leading to the dedifferentiation of mature B lymphocytes into an immature state (lymphoid stem cells). This process initiates the formation of pre-leukaemic cells. Due to the rarity of this chromosomal aberration, only a few cases have been described in the literature. The advantage of this work is the presentation of an interesting case of clonal evolution of cancer cells and the cumulative implications (diagnostic and prognostic) of the patient's genetic alterations.
\end{abstract}

Case presentation: This work presents a patient with diagnosed with TCF3-HLF-positive ALL. Moreover, the additional genetic alterations, which play a key role in the pathogenesis of ALL, were detected in this patient: deletion of a fragment from the long arm of chromosome 13 (13q12.2-q21.1) containing the RB1 gene, intragenic deletions within the PAX5 gene and NOTCH1 intragenic duplication.

Conclusions: A patient with coexistence of chromosomal alterations and the TCF3-HLF fusion has not yet been described. Identifying all these chromosomal aberrations at the time of diagnosis could be sufficient to determine the cumulative effects of the described deletions on the activity of other oncogenes or tumour suppressors, as well as on the clinical course of the disease. On the other hand, complex changes in the patient's karyotype and clonal evolution of cancer cells call into question the effectiveness of experimental therapy.

Keywords: Acute lymphoblastic leukaemia, Case report, TCF3-HLF, Molecular abnormalities, Gene fusion, RB1

\section{Background}

Among childhood leukaemias, B-cell precursor acute lymphoblastic leukaemia (BCP-ALL) has the highest rate of incidence, accounting for approximately $80 \%$ of childhood leukaemias in Western countries [1]. Chromosomal aberrations in ALL can lead to gene fusion, resulting in

\footnotetext{
* Correspondence: lejmanm@poczta.onet.pl; monika.lejman@umlub.pl 'Laboratory of Genetic Diagnostics, Department of Pediatric Hematology, Oncology, and Transplantology, Medical University of Lublin, ul. Antoniego Gębali 6, Lublin, Poland

Full list of author information is available at the end of the article
}

the expression of chimeric fusion proteins. The $t(17$; 19)(q22;p13) translocation leading to the fusion gene TCF3-HLF is a very rare aberration that is probably related to an unfavourable prognosis and is associated with relapse and death within 2 years from diagnosis $[2,3]$.

\section{Case presentation}

A 15-year-old girl was admitted to the Department of Paediatric Haematology, Oncology and Transplantology, Medical University of Lublin, Poland, because of petechiae and bruising on the lower extremities and a pale

(c) The Author(s). 2020 Open Access This article is licensed under a Creative Commons Attribution 4.0 International License, which permits use, sharing, adaptation, distribution and reproduction in any medium or format, as long as you give appropriate credit to the original author(s) and the source, provide a link to the Creative Commons licence, and indicate if changes were made. The images or other third party material in this article are included in the article's Creative Commons licence, unless indicated otherwise in a credit line to the material. If material is not included in the article's Creative Commons licence and your intended use is not permitted by statutory regulation or exceeds the permitted use, you will need to obtain permission directly from the copyright holder. To view a copy of this licence, visit http://creativecommons.org/licenses/by/4.0/ The Creative Commons Public Domain Dedication waiver (http://creativecommons.org/publicdomain/zero/1.0/) applies to the data made available in this article, unless otherwise stated in a credit line to the data. 
complexion. There were no comorbidities, such as obesity, diabetes, or bronchial asthma as well as no significant findings in the patient's family history. The child was diagnosed with common B-cell precursor ALL, and chemotherapy was started in August 2015, according to the ALL IC-BFM 2009 protocol (ALL IntercontinentalBFM 2009). She was classified as being in the intermediate risk group (age $>6$ years, white blood cells $<20,000$ / $\mu \mathrm{l}$; a good response to steroids: on day 8 blast count in peripheral blood <1000/ $\mu$; myelogram on day 15 : $5 \%$ blasts; and minimal residual disease (MRD): $>0.1<10 \%$ ). No central nervous system involvement was observed. According to the ALL IC-BFM 2009 protocol, GTG band staining and fluorescence in situ hybridization (FISH) tests were performed with the use of molecular probes BCR/ABL1, KMT2A and ETV6/RUNX1 (Vysis, Abbot Molecular, Illinois, USA) at the time of diagnosis. The arrangement of signals from the probes used indicated the lack of chromosomal aberrations (Fig. 1). The induction phase of therapy was complicated by poststeroid diabetes and intestinal perforation (a stoma was necessary). This caused a month-long break in chemotherapy. Before continuation of the therapy, the myelogram presented $2 \%$ blasts. The consolidation and reinduction phases of therapy were tolerated well enough. Intensive chemotherapy was completed in June 2016. Maintenance treatment was started in July 2016.
The surgery to close the stoma was performed at the same time.

The patient was readmitted to the clinic in August 2016 due to numerous petechiae, bruising and thrombocytopenia. The bone marrow aspirate smear revealed very early relapse of ALL. Treatment was initiated, according to the IntReALL 2010 HR protocol. Genetic tests were performed again using classical cytogenetics and FISH, and the presence of chromosomal aberrations was again not found, with the exception of an additional signal from chromosome 22 (Fig. 2a). The HIA treatment course applied in weeks 1-4 included dexamethasone, vincristine, methotrexate, PEG-asparaginase, cytarabine, and intrathecal methotrexate. The HIA treatment was complicated by pancreatitis and bradycardia. The myelogram presented $75 \%$ blasts on day 28 . The patient received the HIB treatment course (including dexamethasone, clofarabine, cyclophosphamide, etoposide, intrathecal methotrexate, cytarabine, and prednisolone), but the treatment was unsuccessful. The patient died due to disease progression. Additional analyses of the patient's genetic material are a routine procedure used in patients with the short survival in whom a very aggressive disease course and resistance to treatment were observed. Following the data discussing cases of the patients with a similar course of the disease, we

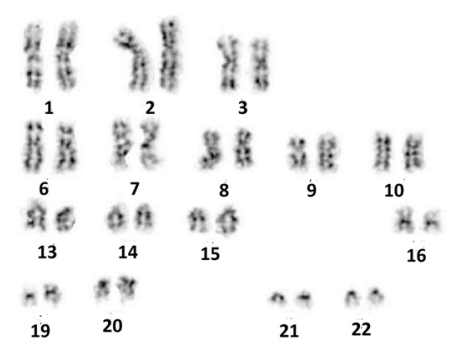

A

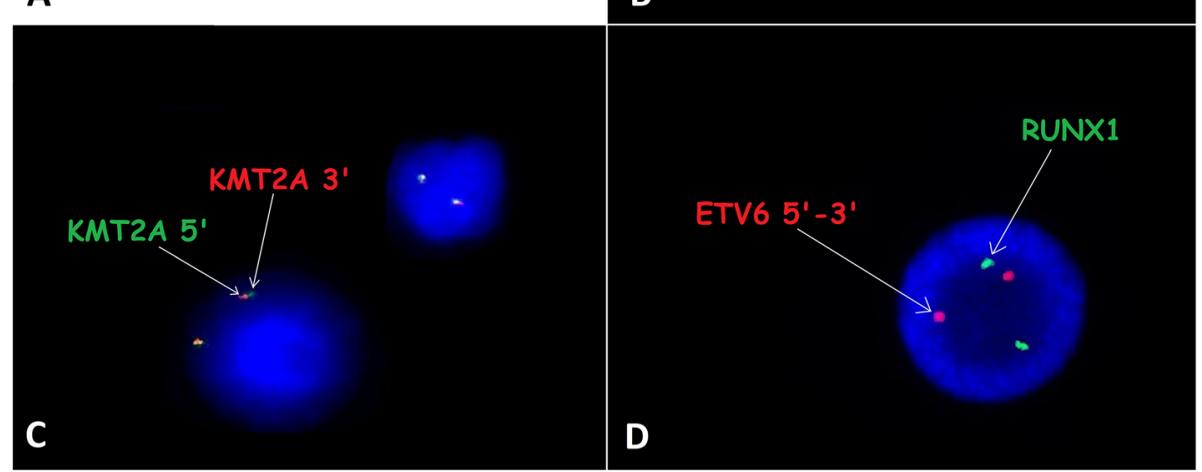

Fig. 1 Cytogenetic features at diagnosis. a GTG band staining study of the patient revealed 46,XX. b, c, d Results of FISH tests with BCR/ABL1, KMT2A and ETV6/RUNX1 probes. FISH was performed on interphase nuclei using probes (Cytocell Ltd., Oxford Gene Technology, Cambridge, United Kingdom) according to the manufacturer's recommendations. Images were captured by an Olympus BX41TF microscope equipped with a Jenoptik camera and analysed with Isis Software (MetaSystems) 




Fig. 2 Cytogenetic features at diagnosis and relapse. a Image of the FISH results with the BCR/ABL1 probe revealing an additional green signal from the BCR locus on chromosome 22. Images of metaphases FISH with the TCF3/PBX/HLF probe in samples from diagnosis (b) and relapse (c). Image of interphase FISH with CCP13/CCP21 probe in samples from diagnosis (d) revealing a lack of 13q deletion genes: MED4, ITM2B, RB1, RCBTB2, and CYSLTR2). FISH was performed on cells in metaphase using probes (Cytocell Ltd., Oxford Gene Technology, Cambridge, United Kingdom) according to the manufacturer's recommendations. Images were captured by an Olympus BX41TF microscope equipped with a Jenoptik camera and analysed with Isis Software (MetaSystems)

used probes for the TCF3-HLF. Retrospective genetic examinations included additional genetic testing (FISH and microarray) of material collected at the time of diagnosis, which showed the presence of the TCF3HLF (Cytocell, Cytocell Ltd., Oxford Gene Technology, Cambridge, United Kingdom) fusion in $89 \%$ of blasts (Fig. 2b and c). Additionally, tests were performed using a CytoScan HD microarray (Applied Biosystems, part of Thermo Fisher Scientific, Waltham, MA) for copy number variation (CNV) analysis, which showed additional changes in the form of a deletion of a fragment of the long arm of chromosome 13 (13q12.2-q21.1) containing the $R B 1$ gene, intragenic deletions within the PAX5 (exons 1-6) gene and NOTCH1 intragenic duplication (exons 3-34). The 13q, PAX5, and NOTCH1 alterations and the TCF3-HLF fusion were present in the same cell clones. The absence of aberrations of $13 \mathrm{q}$ in the karyotype analysis indicates that only cells with a normal karyotype have grown in the cell culture. Deletion of 13q was confirmed in interphase cells by FISH (CCP13/CCP21 FISH Probe Kit, CytoTest). Blasts with $13 \mathrm{q}$ deletion accounted for $86.4 \%$ of the myelogram at initial treatment. Despite molecular studies that revealed a partial $13 \mathrm{q}$ deletion, this aberration was not found in the karyotype analysis (Fig. 1a). Unfortunately, the number of protected samples from the patient was not enough for further tests, including RT-PCR.

Additionally, all the tests mentioned above (GTG band staining, FISH, and microarray) were performed on samples from relapse. The results obtained in samples from relapse suggest the clonal evolution of cancer cells because no 13q deletion was found, while the myelogram presented 77\% blasts with the TCF3-HLF fusion (Fig. 2c and $\mathrm{d})$. Moreover, the microarray test revealed deletion of the CDKN2A and CDKN2B genes (Fig. 3a and b).

The detected translocation $t(17 ; 19)$ is included in the new treatment protocol (AIEOP BFM ALL 2017) as qualifying the patient for the high risk group and for experimental therapy.

\section{Discussion and conclusions}

The described case concerns the translocation $t(17$; 19)(q22;p13), which occurs in $<1 \%$ of BCP-ALL cases $[4,5]$. The presence of this genetic change is associated with very poor prognosis (five-year survival without relapse is 0\%); therefore, it is an independent 


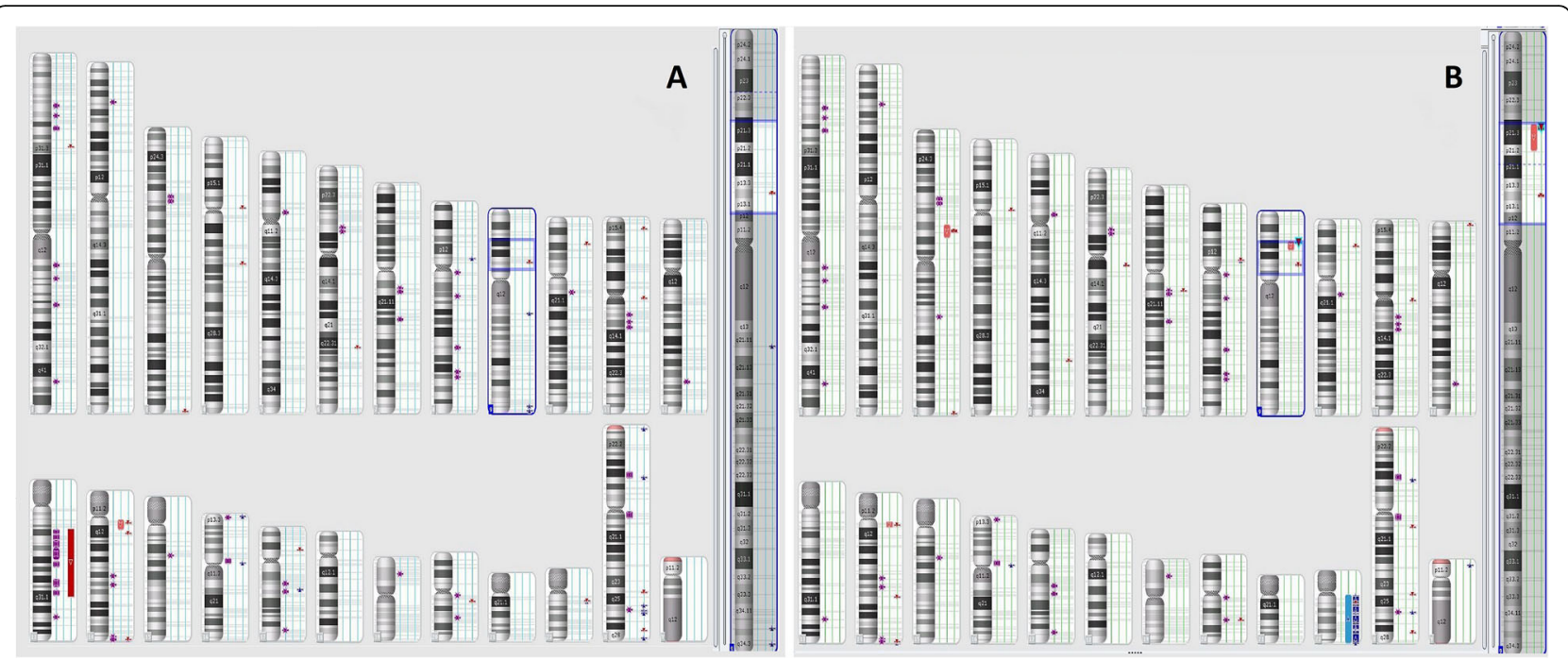

Fig. 3 Karyoviews from microarray results at the time of diagnosis and relapse. a Microarray results revealing a deletion of fragment of the long arm of chromosome 13 (13q12.2-q21.1) containing the RB1 gene (red box), PAX5 intragenic deletion and NOTCH1 intragenic duplication (both on chromosome 9). b Microarray results revealing that $13 q$ deletion was not found in samples from relapse. In addition to the PAX5 and NOTCH1 alterations, CDKN2A/B deletion (red box) and 22 trisomy (blue box) were also observed. Asterisks correspond to deletion (red colour), duplication (blue colour) and loss of heterozygosity (purple colour)

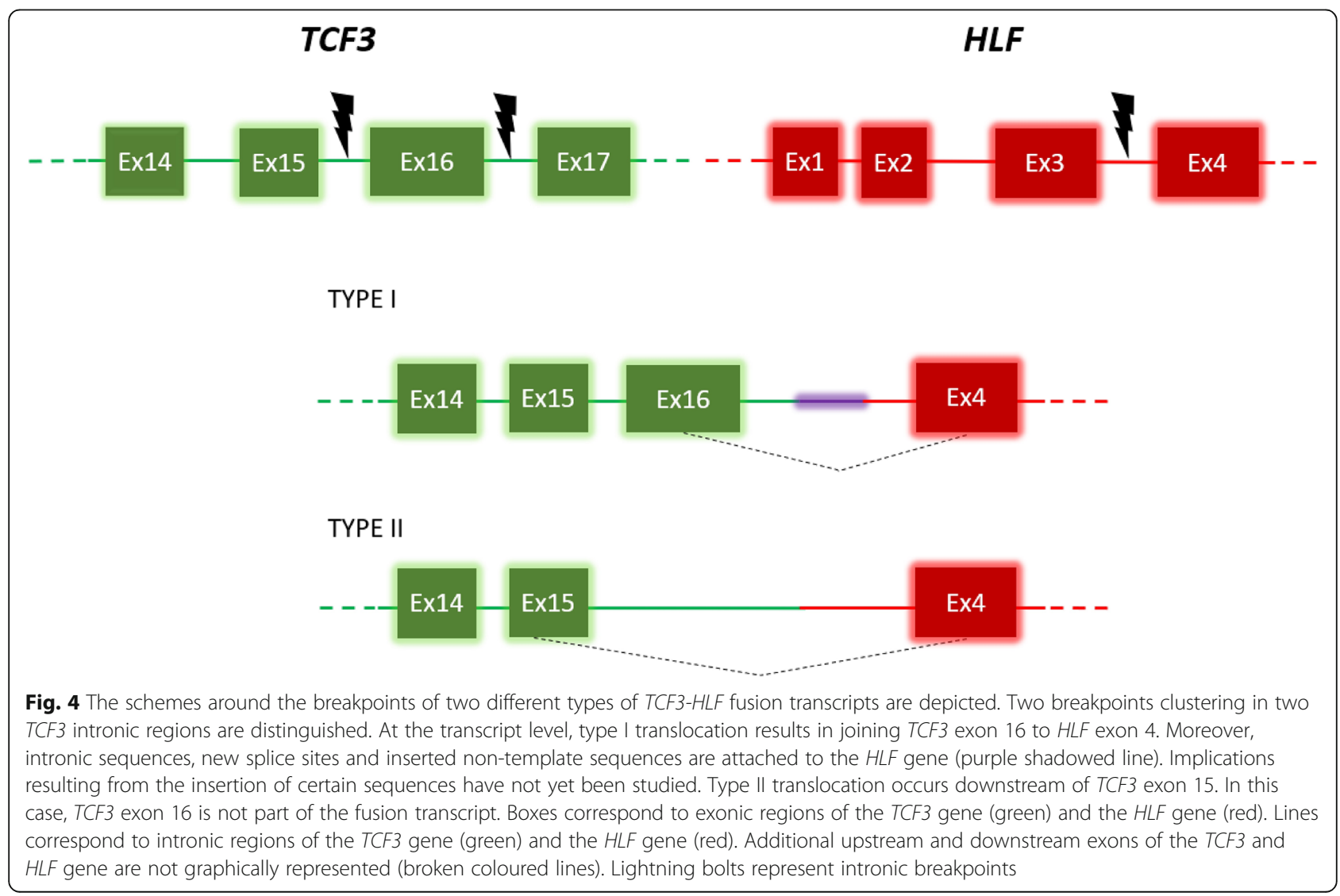


negative prognostic factor. The translocation $t(17 ; 19)$ is associated with poor response to treatment and early relapse of the disease, even after the patient receives haematopoietic stem cell transplantation $[4,6]$. Symptoms associated with $t(17 ; 19)$ include coagulopathy and hypercalcemia [5].

$T C F 3$ is a transcription factor responsible for the maturation of $\mathrm{B}$ lymphocytes; therefore, translocation involving this gene interferes with transcriptional activity throughout the signalling pathways associated with this process $[7,8]$. The $H L F$ gene encodes a bZIP (basic leucine zipper) transcription factor, a member of the proline and acid-rich protein family $[7,9]$.

In our case, the TCF3-HLF fusion was a result of the translocation of the homeobox related gene TCF3 to the leucine zipper domain of $H L F$. TCF3- $H L F$ acts as a transcription factor leading to the dedifferentiation of mature lymphocyte B into an immature state (lymphoid stem cells) (Fig. 4). This process initiates the formation of pre-leukaemic cells $[3,10]$. However, another translocation involving TCF3 is $\mathrm{t}(1 ; 19)(\mathrm{q} 23, \mathrm{p} 13)$. This TCF3-PBX1 fusion accounts for approximately $5-10 \%$ of childhood ALL cases [11]. In this case, PBX1 acts as a transcription factor through fusion of the transcriptional activator domain of TCF3 with $P B X 1$ on $1 \mathrm{p} 23$. In contrast to TCF3-HLF ALL, the 5-year survival rate of TCF3-PBX1 ALL is $80-90 \%$ with intensive chemotherapy $[11,12]$. Differences between both ALL subtypes may be related to the DNA methylation process, which is responsible for the regulation of transcription factors and the structure of chromatin. Over $7000 \mathrm{CpG}$ sites were differentially methylated between TCF3-HLF ALL and TCF3-PBX1 ALL, and these sites correlated with other gene expression levels (including genes involved in leukaemogenesis) [13].

In cases with established TCF3-HLF fusion, mutually exclusive deletions of the PAX5, VPREB1 and BTG1 genes are also observed [3]. Genetic disorders associated with $\mathrm{t}(17 ; 19)$, which also include mutations within the RAS pathway genes (NRAS, KRAS, and PTPN11) may contribute to poor response to treatment [3]. In our case, no changes in any of these genes were observed. However, we found intragenic deletions of PAX5. Expression of the TCF3-HLF fusion gene alone is not sufficient for neoplastic transformation, as implied by studies of TCF3-HLF transgenic mice, which do not develop the human phenotype $[3,14]$.

Despite the rarity of childhood ALL, $t(17 ; 19)$ is being increasingly recognized as a clinically significant chromosomal rearrangement. According to drug screening results, TCF3-HLF-bearing tumour cells obtained from paediatric patients show resistance to standard therapy, including nucleotide analogues (e.g., cytarabine) and mitotic spindle inhibitors (e.g., vincristine) but are sensitive to glucocorticoids [15]. Studies to date show that the use of tyrosine kinase inhibitors or CD19directed immunotherapy can improve the quality of remission in TCF3-HLF-positive ALL patients [16]. Moreover, TCF3-HLF-positive cells are sensitive to PARP inhibitors (olaparib and veliparib) in vitro, but monotherapy with these drugs was not effective in in vivo studies [17].

Moreover, in this case the presence of additional genetic alterations, which play a key role in the pathogenesis of ALL, was detected. Common submicroscopic abnormalities associated with BCP-ALL include alterations within PAX5 - a transcription factor that regulates the normal development and maturation of B-cells. Regardless of which fragment of the PAX5 is deleted, this process results in protein dysfunction [18].

Loss of $13 \mathrm{q}$ is associated with a higher risk of relapse. In this case, due to deletion of the long arm of chromosome 13, the negative cell cycle regulator $R B 1$ was deleted. Loss of function of $R B 1$ due to gene deletion is found in approximately $6 \%$ of BCP-ALL patients. Deletions within $R B 1$ are found in approximately $50 \%$ of all patients who also have 13q loss. The molecular consequences of this recurring deletion are unclear; however, the loss of $R B 1$ is associated with a disruption of the function of other tumour suppressors [19]. In our case, the $R B 1$ deletion was limited to blasts. The karyotype analysis of the patient's parents did not find aberrations of chromosome 13. Deletion of 13q may confer an increased risk of treatment failure. However, to date the deletion of 13q has not been shown to be an independent prognostic indicator $[19,20]$. This case describes loss of $R B 1$ in a patient with TCF3-HLF fusion for the first time.

Abnormalities within the NOTCH1 gene are widely described in T-cell acute lymphoblastic leukaemia ( $\mathrm{T}$ ALL). Alterations within NOTCH1 are characteristic of approximately $50-70 \%$ of T-ALL patients, and most of these abnormalities are NOTCH1 activating mutations. These mutations often result in enhanced signal transduction of the NOTCH1 pathway due to stabilization of the active form of NOTCH1 and impaired degradation of this molecule. Activating mutations of NOTCH1 confer an increased ability to self-renew to cancer cells. Intragenic NOTCH1 deletions account for approximately $2.5 \%$ of all alterations identified in this gene in T-ALL patients [21]. However, there is a lack of reports about the frequency of deletions in NOTCH genes and their effects on the pathogenesis of BCP-ALL. The high percentage of NOTCH1 activating mutations in patients with T-ALL prompted researchers to conduct clinical studies on the effectiveness of the NOTCH1 signalling blockers $\gamma$-secretase inhibitors (GSIs). According to the results obtained so far, the combination of a GSI and 
glucocorticoids in T-ALL therapy may have increased efficacy and decreased toxicity [22]. Moreover, the use of a GSI together with conventional chemotherapy resulted in potentiated drug-induced cell death in B-ALL cells by upregulating intracellular levels of reactive oxygen species [23].

The use of microarrays also allowed for the identification of other unbalanced submicroscopic abnormalities. $C D K N 2 A / B$ deletions are also commonly identified in patients with ALL. The $C D K N 2 A / B$ genes act as tumour suppressors, and monoallelic or biallelic deletions of these genes are often associated with poor survival. Furthermore, the loss of $C D K N 2 A / B$ is often associated with a higher risk of relapse. Deletions of $C D K N 2 A / B$ are often accompanied by a higher WBC count and older patient age at the time of diagnosis. The $C D K N 2 A / B$ and PAX5 deletions are also associated with high-risk cytogenetics, e.g., $B C R-A B L 1$ translocation. Moreover, as a result of the constitutive activation of $N O T C H$ associated with neoplastic transformation, CDKN2A is deleted. Additionally, alterations within $P A X 5$ often correlate with the occurrence of deletions in $C D K N 2 A / B[19,22]$.

Recurrent complex changes in the patient's genotype, including $C D K N 2 A / B$ deletion and $N O T C H 1$ duplication together with maintained $P A X 5$ deletion, may have had an additional impact on the poor outcome and rapid death from disease progression. Furthermore, the clonal evolution of cancer cells with the TCF3-HLF fusion and the genetic alterations described above indicates that experimental treatment might have been ineffective.

A comprehensive analysis of genetic abnormalities may indicate new therapeutic targets, which is of great importance for patients who have not responded appropriately to today's treatment regimens. Due to the rarity of $t(17 ; 19)$, it is difficult to assess the true value of using immunotherapy to improve the outcome of this ALL subtype. TC3F-HLF-positive ALL remains an incurable disease. The most effective therapy in the first line of treatment of high-risk patients remains intensified chemotherapy and haematopoietic stem cell transplantation. Identifying all these chromosomal aberrations at the time of diagnosis could be sufficient to determine the effect of the described deletions on the activity of other oncogenes or tumour suppressors, as well as on the clinical course of the disease. In summary, the described case emphasizes the need to diagnose haematological cancers using high-throughput analytical techniques to obtain a comprehensive picture of genetic alterations.

\section{Abbreviations}

ALL: Acute lymphoblastic leukaemia; HSCT: Haematopoietic stem cell transplantation; BCP-ALL: B-cell precursor acute lymphoblastic leukaemia; MRD: Minimal residual disease; FISH: Fluorescence in situ hybridization; CNV: Copy number variation; T-ALL: T-cell acute lymphoblastic leukaemia;
HIA treatment course: Dexamethasone, vincristine, methotrexate, PEGasparaginase, cytarabine, intrathecal methotrexate; HIB treatment course: Dexamethasone, clofarabine, cyclophosphamide, etoposide, intrathecal methotrexate, cytarabine, prednisolone

\section{Acknowledgements}

Not applicable.

\section{Authors' contributions}

$M L$ and JRK designed the research project. $M L$ and $M W$ are responsible for laboratory work. JZ described clinical data and was responsible for the acquisition of literatures for manuscript. ML and MW prepared final version of manuscript. The final manuscript was reviewed and approved by all authors

\section{Funding}

This work was supported by the National Centre for Research and development, Poland [grant number: STRATEGMED3/304586/5/NCBR/2017]. The funders had no role in study design, data collection, data analysis, interpretation, or writing of the report. The corresponding author had full access to all the data reported in the manuscript and had final responsibility for the decision to submit for publication.

\section{Availability of data and materials}

The datasets generated and/or analysed during the current study are available in the Gene Expression Omnibus (GEO) repository, https://www. ncbi.nlm.nih.gov/geo/query/acc.cgi?acc=GSE147280.

Ethics approval and consent to participate

This study was approved by the ethics committee of Medical University of Lublin, Poland (committee's reference number: KE-0254/178/2002). Written, informed consent to participate was obtained from the patient's parents.

\section{Consent for publication}

Written, informed consent to publish was obtained from the patient's parents.

\section{Competing interests}

The authors declare that they have no competing interests.

\section{Author details}

'Laboratory of Genetic Diagnostics, Department of Pediatric Hematology, Oncology, and Transplantology, Medical University of Lublin, ul. Antoniego Gębali 6, Lublin, Poland. 'Laboratory of Genetic Diagnostics, Medical University of Lublin, Lublin, Poland. ${ }^{3}$ Department of Pediatric Hematology, Oncology, and Transplantology, Medical University of Lublin, Lublin, Poland.

Received: 20 January 2020 Accepted: 24 March 2020

Published online: 03 April 2020

\section{References}

1. Hunger SP, Mullighan CG. Acute lymphoblastic leukemia in children. N Engl J Med. 2015;373:1541-52. https://doi.org/10.1056/nejmra1400972.

2. Harvey RC, Mullighan CG, Wang X, Dobbin KK, Davidson GS, Bedrick EJ, et al. Identification of novel cluster groups in pediatric high-risk B-precursor acute lymphoblastic leukemia with gene expression profiling: correlation with genome-wide DNA copy number alterations, clinical characteristics, and outcome. Blood. 2010;116(23):4874-84. https://doi.org/10.1182/blood2009-08-239681.

3. Fischer U, Forster M, Rinaldi A, Risch T, Sungalee S, Warnatz HJ, et al. Genomics and drug profiling of fatal TCF3-HLF-positive acute lymphoblastic leukemia identifies recurrent mutation patterns and therapeutic options. Nat Genet. 2015;47(9):1020-9. https://doi.org/10.1038/ng.3362.

4. Minson KA, Prasad P, Vear S, Borinstein S, Ho R, Domm J, et al. t(17;19) in children with acute lymphocytic leukemia: a report of 3 cases and a review of the literature. Case Rep Hematol. 2013;2013:563291. https://doi.org/10. $1155 / 2013 / 563291$.

5. Inukai $\mathrm{T}$, Hirose $\mathrm{K}$, Inaba $\mathrm{T}$, Kurosawa $\mathrm{H}$, Hama $\mathrm{A}$, Inada $\mathrm{H}$, et al. Hypercalcemia in childhood acute lymphoblastic leukemia: frequent implication of parathyroid hormone-related peptide and E2A-HLF from translocation 17;19. Leukemia. 2007;21(2):288-96. 
6. Takeda R, Yokoyama K, Ogawa M, Kawamata T, Fukuyama T, Kondoh K, et al. The first case of elderly TCF3-HLF-positive B-cell acute lymphoblastic leukemia. Leuk Lymphoma. 2019;6:1-4. https://doi.org/10.1080/10428194. 2019.1602267.

7. Mullighan CG. Molecular genetics of B-precursor acute lymphoblastic leukemia. J Clin Invest. 2012;122(10):3407-15. https://doi.org/10.1172/ JCl61203.

8. Zhuang $Y$, Soriano $P$, Weintraub $H$. The helix-loop-helix gene E2A is required for B cell formation. Cell. 1994;79(5):875-84. https://doi.org/10.1016/00928674(94)90076-0.

9. Inaba T, et al. Fusion of the leucine zipper gene HLF to the E2A gene in human acute B-lineage leukemia. Science. 1992;257(5069):531-4. https://doi. org/10.1126/science.1386162.

10. Moorman AV. The clinical relevance of chromosomal and genomic abnormalities in B-cell precursoracute lymphoblastic leukaemia. Blood Rev. 2012;26(3):123-35. https://doi.org/10.1016/j.blre.2012.01.001.

11. Felice MS, Gallego MS, Alonso CN, Alfaro EM, Guitter MR, Bernasconi AR, et al. Prognostic impact of $\mathrm{t}(1 ; 19) / \mathrm{TCF} 3-\mathrm{PBX} 1$ in childhood acute lymphoblastic leukemia in the context of Berlin-Frankfurt-Munster-based protocols. Leuk Lymphoma. 2011;52(7):1215-21. https://doi.org/10.3109/ 10428194.2011.565436.

12. Panagopoulos I, Micci F, Thorsen J, Haugom L, Tierens A, Ulvmoen A, et al. A novel TCF3-HLF fusion transcript in acute lymphoblastic leukemia with t(17;19)(q22;p13). Cancer Genet. 2012;205(12):669-72. https://doi.org/10. 1016/j.cancergen.2012.10.004

13. Kahroo P, Szymczak S, Heinsen FA, Forster M, Bethune J, Hemmrich-Stanisak $G$, et al. NGS-based methylation profiling differentiates TCF3-HLF and TCF3PBX1 positive B-cell acute lymphoblastic leukemia. Epigenomics. 2018;10(2): 133-47. https://doi.org/10.2217/epi-2017-0080.

14. Kim M, Choi JE, She CJ, Hwang SM, Shin HY, Ahn HS, et al. PAX5 deletion is common and concurrently occurs with CDKN2A deletion in B-lineageacute lymphoblastic leukemia. Blood Cells Mol Dis. 2011;47(1):62-6. https://doi. org/10.1016/j.bcmd.2011.04.003.

15. Watanabe A, Inukai T, Kagami K, Abe M, Takagi M, Fukushima T, et al. Resistance of t(17;19)-acute lymphoblastic leukemia cell lines to multiagents in induction therapy. Cancer Med. 2019;8(11):5274-88. https://doi.org/10. 1002/cam4.2356.

16. Mouttet B, Vinti L, Ancliff P, Bodmer N, Brethon B, Cario G, et al. Durable remissions in TCF3-HLF positive acute lymphoblastic leukemia with blinatumomab and stem cell transplantation. Haematologica. 2019;104(6): e244-7. https://doi.org/10.3324/haematol.2018.210104.

17. Piao J, Takai S, Kamiya T, Inukai T, Sugita K, Ohyashiki K, et al. Poly (ADPribose) polymerase inhibitors selectively induce cytotoxicity in TCF3-HLFpositive leukemic cells. Cancer Lett. 2017;386:131-40. https://doi.org/10. 1016/j.canlet.2016.11.021.

18. Wrona E, Jakubowska J, Pawlik B, Pastorczak A, Madzio J, Lejman M, et al. Gene expression of ASNS, LGMN and CTSB is elevated in a subgroup of childhood BCP-ALL with PAX5 deletion. Oncol Lett. 2019;18(6):6926-32. https://doi.org/10.3892/ol.2019.11046.

19. Schwab CJ, Chilton L, Morrison H, Jones L, Al-Shehhi H, Erhorn A, et al. Genes commonly deleted in childhood B-cell precursor acute lymphoblastic leukemia: association with cytogenetics and clinical features. Haematologica. 2013;98(7):1081-8. https://doi.org/10.3324/haematol.2013. 085175.

20. Heerema NA, Sather HN, Sensel MG, Lee MK, Hutchinson RJ, Nachman JB, et al. Abnormalities of chromosome bands $13 q 12$ to $13 q 14$ in childhood acute lymphoblastic leukemia. J Clin Oncol. 2000;18(22):3837-44. https://doi. org/10.1200/JCO.2000.18.22.3837.

21. Liu Y, Easton J, Shao Y, Maciaszek J, Wang Z, Wilkinson MR, et al. The genomic landscape of pediatric and young adult T-lineage acute lymphoblastic leukemia. Nat Genet. 2017;49(8):1211-8. https://doi.org/10. 1038/ng.3909

22. Van Vlierberghe $\mathrm{P}$, Ferrando $\mathrm{A}$. The molecular basis of $\mathrm{T}$ cell acute lymphoblastic leukemia. J Clin Invest. 2012;122(10):3398-406. https://doi.org/ 10.1172/JCl61269.

23. Takam Kamga P, Dal Collo G, Midolo M, Adamo A, Delfino P, Mercuri A, et al. Inhibition of Notch signaling enhances chemosensitivity in B-cell precursor lymphoblastic leukemia. Cancer Res. 2019;79(3):639-49. https:// doi.org/10.1158/0008-5472.CAN-18-1617.

\section{Publisher's Note}

Springer Nature remains neutral with regard to jurisdictional claims in published maps and institutional affiliations.

\section{Ready to submit your research? Choose BMC and benefit from:}

- fast, convenient online submission

- thorough peer review by experienced researchers in your field

- rapid publication on acceptance

- support for research data, including large and complex data types

- gold Open Access which fosters wider collaboration and increased citations

- maximum visibility for your research: over $100 \mathrm{M}$ website views per year

At BMC, research is always in progress.

Learn more biomedcentral.com/submissions 